\title{
Superstripes in the Low Energy Physics of Complex Quantum Matter at the Mesoscale
}

\author{
Antonio Bianconi
}

Published online: 21 March 2015

(C) Springer Science+Business Media New York 2015

The most relevant advances on our understanding of the spatial and time fluctuations in heterostructures at an atomic limit have been presented at the Superstripes 2014 conference, held in Erice, Italy, on July 25-31, 2014. A new low energy (in the range between 5 and $250 \mathrm{meV}$ ) physics focusing in the mesoscale (extending in the range between $1 \mathrm{~nm}$ and $100 \mu \mathrm{m}$ ) world is emerging. The intricate mesoscale world driven by quantum critical charge, orbital, and lattice fluctuations is now becoming the main topic of the Superstripes conference series.

This field started in 1992 driven by the interest to understand the nanoscale phase separation of both "electronic matter" and "lattice matter" in cuprate perovskites [1], followed in 1996 by the series of Stripes conferences [2] and in 2008 by the present series of Superstripes conferences [3]. While the majority of the scientific community working in high temperature physics was accepting for 25 years the dogma of a uniform $\mathrm{CuO}_{2}$ plane and a single electronic component, the small scientific community of the Stripes conferences proposed that an intrinsic nontrivial inhomogeneity, with different electronic components and anomalous local lattice fluctuations, was a key feature for the emergence of high temperature superconductivity [3-6]. The complex scenario object of discussions was characterized by a nanoscale phase separation with the competition of different phases in nanoscale domains: (i) short-range spin density wave SDW puddles,; ii) short-range incommensurate charge density wave CDW puddles, and (iii)

\footnotetext{
A. Bianconi $(\bowtie)$

Rome International Center for Materials Science Superstripes, RICMASS, Via dei Sabelli 119/A, 00185, Rome, Italy

e-mail: antonio.bianconi@ricmass.eu
}

superconducting domains. This new scenario, called "superstripes" $[7,8]$ is similar to emulsions in soft matter, where multiple phases at small length scales coexist. The superstripes scenario is today well supported by new experimental methods. The nanoscale phase separation takes place in the mesoscopic world intermediate between the atomic scale (for $d$ less than $1 \mathrm{~nm}$ ) and the macroscopic world (for $d$ larger than $100 \mu \mathrm{m})$. All experiments show that the essential interactions are in the low energy range which is the same range as that of many body interactions determining the emergence of life in the cell.

In materials science, the new low energy physics is determining advances in new nanotechnologies controlling the structure and textures in the mesoscopic scale.

Several research groups have reported at Superstripes 2014 advances on charge density wave physics [9-11]. A new type of light, synchrotron radiation (SR), to investigate the mesoscale matter organization, became available worldwide only in this last 20 years. The invention of the synchrotron radiation source, the storage ring, was made in the 1960s, in Frascati by Bruno Touschek, and the first large synchrotron radiation facilities were developed at Stanford and Frascati in the 1970s. But it was only in these last 20 years that synchrotron radiation was available worldwide and it has allowed to image and control magnetic, lattice, and charge complexity in the mesoscale. The new methodologies probe multiscale spatial and temporal complexity in the low energy range $25-250 \mathrm{meV}$, opening new perspectives for low energy physics. These results are opening new roadmaps toward the design of new room temperature superconductors and new electronic and magnetic functional materials. The XANES and EXAFS methods $[12,13]$ have been applied to investigate the fast local lattice fluctuations of the $\mathrm{Cu}-\mathrm{O}$ bond [14-18], showing that nanoscale phase separation is an essential term in the physics of 
transition metal oxides showing emergent collective quantum phenomena. These results support the idea of Alex Müller where the electron lattice dynamical Jahn-Teller interaction is a key ingredient for the emergence of high temperature superconductivity (HTS). This has been neglected by the majority of the proposed theoretical mechanisms for HTS in cuprates and iron-based superconductors for many years. At Superstripes 2014 advanced SR methods, focusing on X-ray diffraction shows the key role of local lattice distortions in high temperature superconductors and related materials [19-22].

At the Superstripes 2014 conference, complex mesoscopic textures in transition metal oxides, graphene, and silicene have been discussed and the control of the mesoscopic world for the emergence of high temperature superconductivity and electronic functionalities has been presented [23-25]

Advanced theories describing the complex multiphase physics driving the complex nanoscale phase separation have been discussed [26-30]. There is a consensus that the high temperature superconductivity emerges in a landscape where nanoscale incommensurate charge density wave (ICDW) puddles of polaronic ICDW with the associated incommensurate periodic lattice distortions (PLD) in the $\mathrm{CuO}_{2}$ atomic layer coexist with superconducting filamentary domains. The scenario where spin fluctuations mediate high temperature superconductivity has been discussed by Plakida [31], and Jalborg [32] has presented the minimal requirements of band structure electronic features for superconductivity.

The physics of the unconventional superconducting phases made of multicondensates [33] has been the object of many reports [34-37] and of a school on solid state physics held in the previous week.

In conclusion there is agreement that (1) the material architecture of all high temperature superconductors is made of stacks of multiple atomic oxide layers where the active copper oxide, iron, or boron atomic planes are separated by spacer layers; (2) in the active planes, multiple short-range puddles of different electronic phases compete and cooperate; and (3) high temperature superconductivity is a particular case of multigap superconductivity.

\section{References}

1. Müller, K.A., Benedek, G.: "Phase separation in cuprate superconductors" Proceedings of the workshop on phase separation in cuprate superconductors, Erice, Erice, Italy, 6-12 May 1992 (World Scientific, 1993) http://www.worldcat.org/isbn/ 9789810212742
2. Bianconi, A., Saini, N.L.: Saini "Stripes and related phenomena" (Proceedings of the Stripes 1998 conference Rome, Italy) Springer, Selected topics in superconductivity (2000). ISBN 9780-306-47100-1

3. Bianconi, A.: J. Supercond. Nov. Magn. 24, 1117 (2011). doi:10.1007/s10948-011-1142-4

4. Kresin, V., Ovchinnikov, Y., Wolf, S.: Phys. Rep. 431, 231 (2006). doi:10.1016/j.physrep.2006.05.006. http://www.sciencedirect. com/science/article/pii/S0370157306001633

5. Müller, K.A.: J. Supercond. Nov. Magn. 27, 2163-2179 (2014). doi:10.1007/s10948-014-2751-5

6. Phillips, J.C.: J. Supercond. Nov. Magn. 27, 345-347 (2014). doi:10.1007/s10948-013-2308-z

7. Bianconi, A.: Nat. Phys. 9, 536-537 (2013). doi:10.1038/ nphys 2738

8. Bianconi, A.: Int. J. Mod. Phys. B 14, 3289 (2000). doi:10.1142/ S0217979200003769

9. Brazovskii, S.: J. Supercond. Nov. Magn., 1-5 (2014). doi:10.1007/s10948-014-2917-1

10. Yi, T., Bravo, Kirova, N., Brazovskii, S.: J. Supercond. Nov. Magn., 1-5 (2014). doi:10.1007/s10948-014-2916-2

11. Gastiasoro, M.N., Andersen, B.M.: J. Supercond. Nov. Magn., 1-4 (2014). doi:10.1007/s10948-014-2908-2

12. Bianconi, A., Doniach, S., Lublin, D.: Chem. Phys. Lett. 59, 121 (1978). doi:10.1016/0009-2614(78)85629-2

13. Garcia, J., Bianconi, A., Benfatto, M., Natoli, C.R.: Le Journal de Physique Colloques 47, C8 (1986). doi:10.1051/jphyscol:1986807

14. Bianconi, A., Missori, M., Oyanagi, H., Yamaguchi, H., Nishiara, Y., Della Longa, S.: EPL (Europhysics Letters) 31, 411 (1995). doi:10.1209/0295-5075/31/7/012. http://iopscience.iop. org/0295-5075/31/7/012

15. Conradson, S.D., Mustre De Leon, J.J., Bishop, A.R.: J. Supercond. 10, 329 (1997). http://link.springer.com/article/10.1007/ BF02765713\#page-1

16. Mustre de Leon, J., Acosta-Alejandro, M., Conradson, S.D., Bishop, A.R.: J. Synchrotron Radiat. 12, 193 (2005). doi:10.1107/S0909049505000063

17. Mustre de León, J., Acosta-Alejandro, M., Conradson, S.D., Bishop, A.R.: J. Phys. Chem Solids 69, 2288 (2008). doi:10.1016/j.jpcs.2008.04.024

18. de Montellano, G.S.O., Mustre de León, J., pp. 1-5 (2014)

19. Shylin, S., Ksenofontov, V., Medvedev, S., Tsurkan, V., Felser, C.: J. Supercond. Nov. Magn. (2014). doi:10.1007/ s10948-014-2912-6

20. Athauda, A., Yang, J., Li, B., Mizuguchi, Y., Lee, S., Louca, D.: (2014). doi:10.1007/s10948-014-2918-0

21. Ricci, A.: J. Supercond. Nov. Magn. (2014). doi:10.1007/ s10948-014-2907-3

22. Dusza, A., Lucarelli, A., Chu, J.H., Fisher, I.R., Degiorgi, L.: J. Supercond. Nov. Magn. (2014). doi:10.1007/s10948-014-2901-9

23. Poccia, N., Ricci, A., Coneri, F., Stehno, M., Campi, G., Demitri, N., Bais, G., Wang, Hilgenkamp, H.: J. Supercond. Nov. Magn. (2014). doi:10.1007/s10948-014-2902-8

24. Ezawa, M.: J. Supercond. Nov. Magn. (2014). doi: 10.1007/s10948-014-2900-x

25. Bovenzi, N., Finocchiaro, F., Scopigno, N., Bucheli, D., Caprara, S., Seibold, G., Grilli, M.: J. Supercond. Nov. Magn. (2014). doi:10.1007/s10948-014-2903-7

26. Campi, G., Innocenti, D., Bianconi, A.: J. Supercond. Nov. Magn., 1-9 (2015). doi:10.1007/s10948-015-2955-3

27. Carlson, E.W., Liu, S., Phillabaum, B.: J. Supercond. Nov. Magn. (2015). doi:10.1007/s10948-014-2898-0 
28. Saarela, M., Kusmartsev, F.V.: J. Supercond. Nov. Magn. (2015). doi:10.1007/s10948-014-2915-3

29. de Mello, E.V.L.: J. Supercond. Nov. Magn. (2015). doi:10.1007/ s10948-014-2899-z

30. Kapcia, K.: J. Supercond. Nov. Magn. (2014). doi:10.1007/ s10948-014-2906-4

31. Plakida, N.: J. Supercond. Nov. Magn. (2014). doi:10.1007/ s10948-014-2911-7

32. Jarlborg, T.: J. Supercond. Nov. Magn. (2014). doi:10.1007/ s10948-014-2897-1
33. Bianconi, A.: J. Supercond. 18, 625 (2005). doi:10.1007/ s10948-005-0047-5

34. Chávez, I., Grether, M., de Llano, M.: J. Supercond. Nov. Magn. (2014). doi:10.1007/s10948-014-2904-6

35. Yanagisawa, T.: J. Supercond. Nov. Magn. (2014). doi:10.1007/ s10948-014-2905-5

36. Maebashi, H., Takada, Y.: J. Supercond. Nov. Magn. (2014). doi:10.1007/s10948-014-2914-4

37. Croitoru, M.D., Buzdin, A.I.: J. Supercond. Nov. Magn., 1-4 (2014). doi:10.1007/s10948-014-2910-8 